\title{
Transient Ischemic Attack
}

National Cancer Institute

\section{Source}

National Cancer Institute. Transient Ischemic Attack. NCI Thesaurus. Code C50781.

A brief attack (from a few minutes to an hour) of cerebral dysfunction of vascular origin, with no persistent neurological deficit. 